1-6 and 0-2 for periods 1, 2 and 3, respectively. During period 2 only one (10\%) month had zero enrolled participants and three $(30 \%)$ had enrollment of six participants, while around $40 \%$ of the months of periods 1 and 3 , had zero enrolled participants and none had $6(\mathrm{p}=0.004)$. During period 2 and 3, 22 (48\%) participants were enrolled from AS and $6(13 \%)$ from HOC.

Conclusions Active surveillance increased the enrolment of patients with early syphilis, and potentially limited the impact of the COVID-19 pandemic.

\section{P305 HIGH RATES OF SYPHILIS IN BRAZILIAN'S BORDER STRIP: A CHALLENGE TO BE ADDRESSED}

${ }^{1,2} \mathrm{~A}$ Miranda*, 1,2 L Lannoy, ${ }^{2} \mathrm{~A}$ Santos, ${ }^{2} \mathrm{P}$ Gasper, ${ }^{2} \mathrm{R}$ Coelho, ${ }^{2} \mathrm{~N}$ Machado, ${ }^{2} \mathrm{~A}$ Caruso da cunha, ${ }^{2} \mathrm{G}$ Mendes Pereira. ${ }^{1}$ Universidade Federal Do Espirito Santo, Vitoria, Brazil; ${ }^{2}$ Ministry of Health, Brasilia, Brazil

\subsection{6/sextrans-2021-sti.363}

Background Infectious diseases do not recognize international borders. Despite the importance of on the border strip in Brazil, the theme has been little explored under the focus of syphilis data. We aim to analyzing syphilis the detection rate of syphilis in the Brazilian border strip.

Methods A descriptive study including an ecological and crosssectional evaluation employing data from Brazilian Ministry of health databases for 2019. Cases of Syphilis were available at the National System of Disease Notification and diagnostic and treatment data were available at the Primary Health Care System. The syphilis detection rates per 100.000 inhabitants were calculated. Comparisons data were made between the municipalities in the strip border and the federative units.

Results The syphilis detection rate in Brazil in 2019 was 72.8/ 100,000 inhabitants, when the border areas data were analyzed separately the detection rate in the country was 71.6/ 100,000 and in the border region 91.9/100,000. In the bordering regions, the highest detection rate was observed in the southern region (116.0/100,000 inhabitants), and the lowest in the northern region (55.6/1000,000). Brazil had 152,915 cases of acquired syphilis, of which 10,636 (6.9\%) were in border areas. A total of $20 \%$ of the municipalities had no cases of syphilis and $31.6 \%$ had a detection rate above the national average. There are 263(45\%) municipalities up to 7,000 inhabitants, $82 \%$ up to 25,000 inhabitants and only $11(2 \%)$ above 100,000 inhabitants.

Conclusions Brazil has a high detection rate of syphilis and this rate is even higher when it was considered the strip border in general. Nevertheless, the rates were different when it was compared the borders with different countries. There is a need to improve the countries' capacity to collect high quality data in the coverage of interventions and inequalities and to use this data as a basis for decisions to improve care.

\section{P306 PUBLIC POLICIES FOR SYPHILIS IN PREGNANCY AND CONGENITAL SYPHILIS IN BRAZILIAN BORDER STRIP}

${ }^{1,2} \mathrm{~L}$ Lannoy, ${ }^{2} \mathrm{~A}$ Santos, ${ }^{2} \mathrm{P}$ Gaspar, ${ }^{2} \mathrm{R}$ Coelho, ${ }^{2} \mathrm{~A}$ Guarabyra, ${ }^{2} \mathrm{~A}$ Caruso da Cunha, ${ }^{2} \mathrm{G}$ Mendes Pereira, ${ }^{1,2} \mathrm{~A}$ Miranda*. 'Universidade Federal Do Espirito Santo, Vitoria, Brazil; ${ }^{2}$ Ministry of Health, Brasilia, Brazil

10.1136/sextrans-2021-sti.364
Background Congenital syphilis (CS) is a public health issue in Brazil. Despite the importance of on the border strip, the country do not have many data in these areas. Our goal is analyzing the profile of syphilis in pregnancy and CS in the Brazilian border strip.

Methods We performed an ecological and cross-sectional study using data from 2019. Cases of Syphilis were available at the National System of Disease Notification and diagnostic and treatment data were available at the Primary Health Care System. The syphilis in pregnancy and CS detection rates per 1.000 living-births (LB) were calculated. Comparisons data were made between the municipalities in the strip border and the federative units.

Results Among the 586 municipalities, $84.8 \%$ carry out rapid test, 391(67\%) reported syphilis in pregnancy and 396(67.6\%) applied benzathine penicillin in primary care units. Regarding CS, 366(64\%) municipalities did not report CS cases, 90 (17\%) had a lower incidence than the national average and $102(19 \%)$ had similar or greater incidence. Around $45 \%$ of women were diagnosed in the first trimester of pregnancy; this proportion was higher in the southern region $(52.6 \%)$. Around $80.5 \%$ of women received adequate treatment, with no difference between regions. The national detection rate of syphilis during pregnancy was 20.8 cases/1000LB and in border region was 23.3/1000LB. Regarding the CS incidence, we calculated 8.2/1000LB for Brazil and 5.5/1000LB for border region. It is noted that the northern region presented the lowest syphilis rate in pregnancy $(19.2 / 1000 \mathrm{NV})$ and CS (3.7/ 1000LB) compared to the Midwest (27.9/1000LB and 6,4/ 1000LB, respectively) and South (24.9/1000LB and 6.6/ 1000LB, respectively).

Conclusion The results showed a good performance of care in pregnancy and low rate of CS. Although it is still important diagnostic and treatment implementation to qualify the care in order to reduce the vertical transmission of syphilis in the Brazilian border strip.

\section{P310 PREFERENCES FOR CHLAMYDIA TESTING AND MANAGEMENT IN HONG KONG: A DISCRETE CHOICE EXPERIMENT}

${ }^{1} \mathrm{~J}$ Ong ${ }^{*},{ }^{1} \mathrm{C}$ Fairley, ${ }^{2} \mathrm{~J}$ Hocking, ${ }^{3} \mathrm{~K}$ Turner, ${ }^{3} \mathrm{R}$ Booton, ${ }^{4} \mathrm{D}$ Tse, ${ }^{4} \mathrm{~W}$ Wong. ${ }^{1}$ Melbourne Sexual Health Centre, 580 Swanston Street, Australia; ${ }^{2}$ The University of Melbourne, Parkville, Australia; ${ }^{3}$ University of Bristol, Bristol, UK; ${ }^{4}$ The University of Hong Kong, Hong Kong

\subsection{6/sextrans-2021-sti.365}

Background As most chlamydia cases are asymptomatic, regular screening and timely management is important for chlamydia control. We aimed to determine the preferences of people living in Hong Kong for chlamydia testing and management services.

Methods An online panel (hosted by Toluna) of sexually active individuals living in Hong Kong completed an online survey with two discrete choice experiments (DCEs). The first DCE examined the preferred attributes of a chlamydia testing service (cost, location, appointment time, speed of results, delivery of results and availability of other STI testing). The second DCE examined the preferred attributes of a chlamydia management service (cost, access to patient delivered partner therapy (PDPT), location, travel time, type of person consulted, and attitude of staff). 
Results A representative sample of 520 individuals participated; average age was 36.8 years (SD 9.9), 40\% were males and $66 \%$ had a Bachelor's degree or higher. The choice to test was most influenced by cost (free), followed by speed of results (in 14 days), delivery of results (via SMS), extra STI testing, appointment available (same day), and the least important was location of testing (private hospital). The choice to attend for management of chlamydia was most influenced by the attitude of staff (not rude), followed by cost (free), who they consult (specialist), access to PDPT, travel time (less than 30 minutes) and the least important was treatment location (antibiotics sent to home). There was significant heterogeneity in preferences related to age, place of birth, those reporting more than one sexual partner in the preceding six months and gender.

Conclusion To design effective chlamydia testing and management services, it is important to account for patient preferences. For people living in Hong Kong, cost and staff attitude were the most important factors for deciding whether to test or be managed for chlamydia, respectively.

\section{P311 ASSOCIATION BETWEEN CHOICE OF NON-BARRIER CONTRACEPTIVE AND CONDOM USE AMONG FSWS IN CHINA: IMPLICATIONS FROM A NATIONAL CROSS- SECTIONAL STUDY}

1,2,3Y Wang*, ${ }^{4} \mathrm{~K}$ Dooley, ${ }^{5} \mathrm{~W}$ Zhang, ${ }^{4} \mathrm{~K}$ Smith, ${ }^{1,2,3} \mathrm{C}$ Wang. ${ }^{1}$ Dermatology Hospital of Southern Medical University, Guangzhou, China; ${ }^{2}$ Southern Medical University Institute for Global Health and Sexually Transmitted Diseases, Guangzhou, China; ${ }^{3}$ Guangdong Provincial Center for Skin Disease and STI Control, Guangzhou, China; ${ }^{4}$ Division of Epidemiology and Community Health, University of Minnesota Twin Cities, Minneapolis, USA; ${ }^{5}$ University of North Carolina at Chapel Hill, Project-China, Guangzhou, China

\subsection{6/sextrans-2021-sti.366}

Background Condom promotion was primarily to prevent human immunodeficiency virus (HIV) among Chinese female sex workers (FSWs), which may have missed to improve their reproductive health and sexual health holistically. A more complete understanding of FSWs' sexual and reproductive health needs specifically pertaining to the choice of contraceptive would be beneficial to make progress in disease control. This study aimed to better characterize contraceptive use and explore the interaction between contraception and condom use among Chinese FSWs.

Methods A cross-sectional study was conducted in eight cities throughout 7 provinces in China, 2019. Participants completed a survey including sociodemographic information, sexual behaviors and reproduction measures. The exposure of interest was non-barrier contraceptive use, and the outcome of interest was inconsistent condom use with clients. Multivariate logistic regression and subgroup analysis were conducted to assess the relationship between non-barrier contraceptive use and condom use among Chinese FSWs.

Results In total, 1229 FSWs participated in the study with a median age of 35 years. 629(51.2\%) women reported using non-barrier contraceptives while 586 (47.7\%) used barrier contraceptives. Odds of inconsistent condom use was higher $(\mathrm{aOR}=1.24$, 95\% CI: 0.99-1.57) among nonbarrier contraceptives users than among barrier contraceptives users, but it lacks statistical significance. However, for women in middle-tier venues $(\mathrm{aOR}=0.66,95 \% \mathrm{CI}$ : 0.46-0.96), upper-tier venues $(\mathrm{aOR}=1.64$, 95\% CI: 1.14 2.36), and those who completed senior high school or above (aOR $=4.07,95 \%$ CI: 2.20-7.52), we observed correlation between inconsistent condom use and non-barrier contraception.

Conclusion We observed that non-barrier contraceptive users who work in upper tier settings and with higher educational attainment tend to use condom inconsistently. Condom promotion efforts currently focused on HIV and STI prevention among FSWs in China would benefit from considering the contraceptive and reproductive needs of this population.

\section{P312 DESIGNING HIV TESTING AND SELF-TESTING SERVICES FOR YOUNG PEOPLE IN NIGERIA: A DISCRETE CHOICE EXPERIMENT}

${ }^{1} \mathrm{~J}$ Ong ${ }^{*},{ }^{2} \mathrm{U}$ Nwaozuru, ${ }^{2} \mathrm{C}$ Obiezu-Umeh, ${ }^{3} \mathrm{C}$ Airhihenbuwa, ${ }^{2} \mathrm{H}$ Xian, ${ }^{4} \mathrm{~F}$ Terris-Presholt, ${ }^{5} \mathrm{~T}$ Gbajabiamila, ${ }^{5} \mathrm{D}$ Oladele, ${ }^{5} \mathrm{I}$ Idigbe, ${ }^{5} \mathrm{~A}$ David, ${ }^{5} \mathrm{~J}$ Okwuzu, ${ }^{5} \mathrm{~T}$ Bamidele, ${ }^{2} \mathrm{~J}$ Iwelunmor, ${ }^{6}$ J Tucker, ${ }^{5} 0$ Ezechi. ${ }^{1}$ Melbourne Sexual Health Centre; ${ }^{5} 80$ Swanston Street, Australia; ${ }^{2}$ Saint Louis University College for Public Health and Social Justice, USA; ${ }^{3}$ Georgia State University, Atlanta, USA; ${ }^{4}$ UNAIDS, Switzerland; ${ }^{5}$ The Nigerian Institute of Medical Research, Lagos, Nigeria; ${ }^{6}$ London School of Hygiene and Tropical Medicine, London, UK

\subsection{6/sextrans-2021-sti.367}

Introduction A third of new HIV infections occur among young people and the majority of young people living with HIV are in sub-Saharan Africa. Global scale-up of HIV selftesting (HIVST) could improve testing rates among young people in SSA, who have reported suboptimal HIV testing. We examined the strength of Nigerian youth preferences related to HIV testing.

Methods Discrete choice experiments (DCEs) were conducted among Nigerian youth (age 14-24 years). Participants completed one of two DCEs: one for preferred qualities of HIV testing (cost, location of the test, type of test, the person who conducts the test, and availability of HIV medicine at the testing site) and another for preferred qualities of HIVST kits (cost, test quality, type of test, extra items and support if tested positive)

We use random parameters logit model to quantify the relative strength of preferences and test for preference heterogeneity.

Results A total of 504 youth participated: mean age 21 (SD 2) years, $38 \%$ were men, and $35 \%$ had higher than secondary school education. Youth equally preferred HIV testing services in public hospitals and home testing, all other attributes being equal. For HIVST kits, youth preferred the kit to be low cost (up to \$USD1.38), accessible from community health centers, and integrated with self-testing for syphilis and other sexually transmitted infections (STIs). Preferences differed according to age, education level, gender, sexual, and HIV testing behaviours.

Conclusions We found that there could be demand for HIVST for Nigerian youth, who preferred HIVST kits that integrate testing for other STIs and is accessed from community health centers. These data could inform tailoring of HIV testing delivery services for Nigerian youth, to ultimately increase reach and acceptability. 